\title{
Relationships Among Depression, Anxiety, and Insomnia Symptoms in Perinatal Women Seeking Mental Health Treatment
}

\author{
Leslie M. Swanson, Ph.D., Scott M. Pickett, Ph.D., Heather Flynn, Ph.D., and Roseanne Armitage, Ph.D.
}

\begin{abstract}
Background: Depression and anxiety symptoms are commonly experienced by women during the perinatal period. Changes in sleep and sleep quality are typical throughout pregnancy and early postpartum. However, little is known about relationships between insomnia symptoms and psychiatric symptoms in perinatal women. The objective of the present study is to characterize the burden of insomnia symptoms in perinatal women seeking outpatient psychiatric treatment and to examine relationships between insomnia and symptoms of depression and anxiety.

Methods: Data from 257 pregnant or postpartum women who sought outpatient psychiatric treatment at a university hospital-affiliated clinic were extracted from an existing clinical management database. Data included validated self-report measures assessing insomnia (Insomnia Severity Index [ISI]), mood (Edinburgh Postnatal Depression Scale [EPDS]), and generalized anxiety (Penn State Worry Questionnaire [PSWQ]).

Results: Fifty-two percent of women reported symptoms of insomnia, 75\% reported symptoms of depression, and $61 \%$ reported symptoms of generalized anxiety. After controlling for PSWQ, the partial correlation between EPDS and ISI was 0.15 and 0.37 for pregnant and postpartum women, respectively. After controlling for EPDS, the partial correlation between PSWQ and ISI was 0.20 and 0.12 for pregnant and postpartum women, respectively. Women with clinically significant ISI scores had significantly higher odds for reporting symptoms consistent with depression (odds ratio [OR] 7.7) and generalized anxiety (OR 2.55) compared to women with lower ISI scores.

Conclusions: Insomnia symptoms affected a significant proportion of the perinatal women in this sample. These symptoms are linked to symptoms of depression and anxiety in treatment-seeking pregnant and postpartum women. Perinatal women seen in psychiatric treatment settings should be routinely screened for sleep problems.
\end{abstract}

\section{Introduction}

D EPRESSION IS EXPERIENCED by many women during the perinatal period and is widely considered to be the most common and debilitating complication of childbirth. ${ }^{1,2}$ Perinatal depression affects 1 in 5 women, whereas anxiety disorders, although less studied than depression, are estimated to impact as many as $16 \%$ of women in the early postpartum period. ${ }^{3,4}$ Impaired maternal functioning due to mental illness can affect bonding between mother and infant and have longer-term consequences for offspring, including developmental and cognitive impairments, self-regulation difficulties, low self-esteem, and behavior problems. $^{5-7}$
Women experience a myriad of changes during pregnancy and postpartum that may increase their vulnerability to affective illness, including hormonal fluctuations, increasing psychosocial demands, and dysregulation of the sleep-wake cycle. Alterations in sleep patterns are seen as early as the first trimester, when slow-wave sleep decreases and does not recover until after delivery. ${ }^{8}$ Total sleep time and sleep efficiency decline throughout pregnancy and reach a nadir within the first month postpartum. ${ }^{8,9}$ Sleep disruption and fragmentation of nighttime sleep are extremely common in healthy pregnant and postpartum women. ${ }^{8,10}$ However, the sleep changes during pregnancy and the sleep deprivation that is common early in the postpartum period may play a role in risk for postpartum depression.

\footnotetext{
${ }^{1}$ Department of Psychiatry, University of Michigan, Ann Arbor, Michigan.

${ }^{2}$ Department of Psychology, Oakland University, Rochester, Michigan.
} 
The existing literature has documented associations between sleep disruptions and mood problems during the perinatal period. Subjectively reported sleep quality was a significant predictor of depressive symptoms in postpartum women at low risk for depression, although a weaker relationship was found between objective sleep measures and mood. ${ }^{9}$ In their study of 124 women from the third trimester of pregnancy to 3 months postpartum, Goyal et al. ${ }^{11}$ found that subjective sleep disturbance was associated with depressive symptoms at the third trimester and at the third month postpartum, with delayed sleep onset particularly salient to depressive symptoms. In a prospective study of 273 pregnant women surveyed at three time points from the second trimester through the third trimester, subjectively reported sleep quality around 18 weeks and 26 weeks of gestation was a significant predictor of depressive symptoms later in pregnancy; notably, depressive symptoms earlier in pregnancy were not related to sleep quality at later time points. ${ }^{12}$ Similarly, in a Norwegian population-based study of $>4000$ women at 7 weeks postpartum, subjective report of poor sleep quality was significantly associated with depression even after controlling for multiple risk factors. ${ }^{13}$ Poor sleep quality in the first 17 weeks after delivery increased the risk for depression recurrence in women who had a past history of major depressive disorder (MDD) but were not depressed during pregnancy. ${ }^{14}$

Most published studies of sleep in perinatal women using subjective assessments of sleep have focused on sleep quality. Although it is important to study sleep quality, there are other aspects of sleep, such as insomnia symptoms, that remain understudied in perinatal women. (The term "insomnia symptoms" is used to characterize complaints of sleep disruption associated with sleep loss but is not diagnostic of clinical insomnia. Further, the reasons for sleep loss may be multifactorial in this population, for example, attending to the infant, physical discomfort, physiological changes, or difficulty falling asleep because of conditioned arousal.) The perinatal period is rife with factors that disrupt sleep and may precipitate and perpetuate insomnia, including the birth of a child, caregiving demands, napping, and inconsistent sleepwake schedules, ${ }^{15,16}$ yet little is known about the influence of sleep disruption on mood and functioning around the time of childbearing. It is important to distinguish between the sleep disruption experienced by most women during pregnancy and early postpartum and insomnia. Insomnia is characterized by subjectively reported difficulty falling asleep, maintaining sleep, or poor sleep quality, accompanied by distress and functional impairments. Given the sleep fragmentation and chronic sleep loss that most pregnant and postpartum women experience, they should be able to fall asleep quickly. Thus, it has been suggested that insomnia in childbearing women may occur as difficulty falling asleep or returning to sleep in the absence of physical discomfort or environmental disturbances (e.g., noise or demands from the infant). ${ }^{17,18}$ Insomnia has been found to independently increase the risk for depression and anxiety disorders in nonperinatal populations. ${ }^{19,20}$ To date, there are no studies of the relationships between insomnia symptoms and psychiatric symptoms in perinatal women who are seeking mental health treatment. Thus, it remains unknown if insomnia symptoms increase the risk for mental illness or if insomnia symptoms comorbid with anxiety and depression worsen the clinical course in perinatal women. Research clarifying these relationships can inform interventions targeting sleep disturbance to enhance treatment outcomes.

The aims of the present study are to describe the burden of insomnia symptoms in perinatal women seeking outpatient psychiatric treatment and examine associations between insomnia symptoms and psychiatric symptoms (i.e., symptoms of depression and anxiety) in this population. Specifically, we were interested in whether symptom severity or rates of insomnia symptoms differ between pregnant and postpartum women, which insomnia symptoms best predict depression and anxiety symptom severity, and if experiencing more severe symptoms of insomnia increases the likelihood of experiencing depression and anxiety symptoms. We hypothesized that compared to pregnant women, postpartum women would have higher insomnia symptom severity scores, and more postpartum women would meet the threshold for clinically significant insomnia symptoms. We also expected that experiencing significant symptoms of insomnia would be associated with increased risk for depression and anxiety symptoms.

\section{Materials and Methods}

\section{Participants}

Participants were 257 pregnant $(n=114)$ or postpartum $(n=143)$ women who sought evaluation and treatment at a university hospital-affiliated outpatient psychiatry clinic. Women were classified as postpartum if they had given birth in the 6 months prior to their intake visit. Participants ranged in age from 19 to 51 years of age, with a mean age of $31 \pm 6$ years.

\section{Procedures}

All patients who come to the clinic for an intake visit complete assessments of mood and sleep as part of routine care in the clinic. The data from these assessments are maintained in a clinical management database. It is from this source that we extracted the data for the present study. The data were de-identified, and the study was granted an exemption from institutional board review by the University of Michigan Medical Institutional Review Board.

\section{Measures}

Participants completed the Insomnia Severity Index (ISI), the Edinburgh Postnatal Depression Scale (EPDS), and the Penn State Worry Questionnaire (PSWQ). The ISI is a 7-item self-report measure of severity of insomnia symptoms over the past 2 weeks. It has good reliability and validity. ${ }^{21}$ Scores on this measure range from 0 to 28 . Scores between 0 and 14 indicate mild insomnia symptoms, scores between 15 and 21 indicate insomnia symptoms of moderate severity, and scores $\geq 22$ indicate severe insomnia symptoms.

The EPDS 22 is a valid, reliable 10-item self-report depression screening questionnaire that screens for depressive symptoms over the past 7 days in pregnant and postpartum women. EPDS scores range from 0 to 30, with higher scores indicating more symptoms of depression. Cox et al. ${ }^{23}$ suggest a clinical cutoff of 12 or 13 for significant depressive symptoms, which has a sensitivity of $81 \%$ and a positive predictive value of $24 \%$ for detecting MDD as diagnosed using Research 
Table 1. Means and Percentages of Women Above Clinical Threshold for Edinburgh Postnatal Depression Scale, Penn State Worry Questionnaire, and Insomnia Severity Index

\begin{tabular}{|c|c|c|c|c|c|c|}
\hline & \multicolumn{2}{|c|}{ Pregnant $\mathrm{n}=114$} & \multicolumn{2}{|c|}{ Postpartum $\mathrm{n}=143$} & \multicolumn{2}{|c|}{ Total sample $\mathrm{n}=257$} \\
\hline & Mean $\pm S D$ & $\%$ above threshold & Mean $\pm S D$ & $\%$ above threshold & Mean $\pm S D$ & $\%$ above threshold \\
\hline EPDS & $15.81 \pm 6.29$ & 68 & $16.94 \pm 5.08$ & 80 & $16.44 \pm 5.67$ & 75 \\
\hline PSWQ & $59.35 \pm 19.97$ & 54 & $64.39 \pm 16.46$ & 66 & $62.17 \pm 18.23$ & 61 \\
\hline ISI & $14.04 \pm 6.41$ & 45 & $14.48 \pm 6.05$ & 55 & $14.28 \pm 6.21$ & 52 \\
\hline
\end{tabular}

EPDS, Edinburgh Postnatal Depression Scale; ISI, Insomnia Severity Index; PSWQ, Penn State Worry Questionnaire; SD, standard deviation.

Diagnostic Criteria. Thus, participants scoring $>12$ on this measure were classified as experiencing clinically significant symptoms of depression.

The PSWQ ${ }^{24}$ is a 16-item self-report measure designed to assess worry and symptoms characteristic of generalized anxiety disorder. It has good validity and reliability. ${ }^{24}$ Scores on this measure range from 16 to 80 . Participants scoring $\geq 60$ on the measure were classified as experiencing generalized anxiety symptoms.

\section{Statistical analyses}

All statistical analyses were performed using SPSS version 17 (SPSS, Inc., Chicago, IL). $t$ Tests with Bonferroni corrections compared differences in mean scores for the ISI, EPDS, and PSWQ between pregnant and postpartum women, and chisquare analyses with Bonferroni corrections tested whether the proportions of pregnant and postpartum women meeting the cutoff scores for the ISI, EPDS, and PSWQ were different. Correlations and partial correlations were computed between total scores on the ISI, EPDS, and PSWQ. Linear regression analyses examined the contribution of each ISI item to EPDS and PSWQ total scores. Odds ratios (ORs) were calculated using logistic regression to determine if women reporting moderate or severe symptoms of insomnia were at higher risk for scoring above cutoffs on the EPDS and PSWQ compared to women who had subthreshold insomnia symptoms. Regression analyses were adjusted for maternal age because age was associated with EPDS and PSWQ scores. Cohen's $d$ for differences in mean ISI score between those women above and below the clinical thresholds for the EPDS and PSWQ was also computed. The EPDS contains one item assessing for sleep problems, and analyses were conducted with and without that item. No significant difference was observed in the results, so the results reported here use all EPDS items to calculate total score.

\section{Results}

Chronbach alpha values indicated good internal consistency in this sample for all the measures $(\mathrm{EPDS}=0.85, \mathrm{ISI}=$ 0.87 , PSWQ $=0.95$ ). Mean values and percentage of participants scoring above the clinical threshold for the ISI, PSWQ, and EPDS are presented in Table 1 for the total sample and for the subsamples of pregnant and postpartum women. A significant proportion of women scored above the clinical cutoffs for all the measures. There was a very high incidence of women meeting the clinical cutoffs for depressive symptoms, generalized anxiety symptoms, and insomnia symptoms, particularly among postpartum women. Approximately $12 \%$ of pregnant women and $11 \%$ of postpartum women $(12 \%$ of the total sample) scored above the threshold for severe insomnia. Mean EPDS $(\mathrm{t}(255)=-1.60, p=0.357)$, PSWQ $(\mathrm{t}(254)=-2.21, p=0.084)$, and ISI $(\mathrm{t}(247)=-0.56, p=1.0)$ scores were not significantly different between pregnant and postpartum women. Similarly, proportions of women scoring above the clinical thresholds were not different between pregnant and postpartum women for the EPDS (chi-square (1, $n=256)=5.07, p=0.072)$, ISI (chi-square $(1, n=249)=2.76$, $p=0.291$ ), or PSWQ (chi-square $(1, n=256)=4.11, p=0.129)$.

The correlations for the total sample, pregnant women, and postpartum women are shown in Table 2. The EPDS and PSWQ correlated significantly with the ISI in the total sample and in each group separately. As all three measures were correlated in the total sample and in each group, we also computed partial correlations to remove the overlapping influence of EPDS and PSWQ. The resultant partial correlations are shown in Table 2.

After removing the influence of PSWQ, the correlation between EPDS and ISI remained significant in postpartum women but not in pregnant women, indicating that the relationship between depression symptoms and insomnia symptoms was significantly influenced by generalized anxiety symptoms, but only in pregnant women. The relationship between depression symptoms and insomnia symptoms was not influenced by generalized anxiety symptoms in women in the postpartum period. However, EPDS also affected the relationship between PSWQ and ISI. Removing its influence greatly decreased the relationship between generalized anxiety symptoms and insomnia symptoms in both pregnant and postpartum women, from $25 \%$ to $4 \%$ of the explained

\section{Table 2. Bivariate and Partial Correlations Among Insomnia Severity Index, Edinburgh Postnatal Depression Scale, and Penn State Worry Questionnaire}

\begin{tabular}{llcc}
\hline & $\begin{array}{c}\text { Total sample } \\
\mathrm{n}=257\end{array}$ & $\begin{array}{c}\text { Pregnant } \\
\mathrm{n}=114\end{array}$ & $\begin{array}{c}\text { Postpartum } \\
\mathrm{n}=143\end{array}$ \\
\hline EPDS with PSWQ & $0.69^{* * *}$ & $0.81^{* * *}$ & $0.53^{* * *}$ \\
$\quad$ Controlling for & $0.63^{* * *}$ & $0.78^{* * *}$ & $0.46^{* * *}$ \\
$\quad$ ISI & & & \\
EPDS with ISI & $0.49^{* * *}$ & $0.50^{* * *}$ & $0.48^{* * *}$ \\
Controlling for & $0.29^{* * *}$ & 0.15 & $0.37^{* * *}$ \\
$\quad$ PSWQ & & & \\
PSWQ with ISI & $0.43^{* * *}$ & $0.51^{* * *}$ & $0.53^{* * *}$ \\
$\quad$ Controlling for & $0.14^{*}$ & $0.20^{*}$ & 0.12 \\
$\quad$ EPDS & & & \\
\hline
\end{tabular}

${ }^{*} p<0.05 ; * * * p \leq 0.001$. 
Table 3. Insomnia Severity Index Items as Predictors of Edinburgh Postnatal Depression Scale TOtAL SCORE

\begin{tabular}{lrrrr}
\hline & $B$ & $S E B$ & $\beta$ & \multicolumn{1}{c}{$\mathrm{p}$} \\
\hline Maternal age & -0.09 & 0.06 & -0.09 & 0.076 \\
Difficulty falling asleep & 1.36 & 0.35 & 0.28 & $<0.001$ \\
Difficulty staying asleep & 0.35 & 0.39 & 0.07 & 0.383 \\
Problem waking too early & 0.35 & 0.30 & 0.08 & 0.245 \\
Dissatisfaction with sleep pattern & -0.53 & 0.47 & -0.09 & 0.253 \\
Daytime functioning impaired & 0.27 & 0.43 & 0.06 & 0.539 \\
Impairment noticeable to others & 0.67 & 0.35 & 0.15 & 0.056 \\
Distress about sleep problem & 0.55 & 0.41 & 0.12 & 0.187 \\
\hline
\end{tabular}

B, unstandardized coefficient; SE B, standard error of unstandardized coefficient.

variance. Thus, the interrelationship among depression, insomnia, and generalized anxiety symptoms differed between pregnant and postpartum women.

To determine which specific insomnia symptoms were cross-sectional predictors of EPDS and PSWQ total score, linear regressions were computed, removing the influence of maternal age first. These results are shown in Tables 3 and 4 for the EPDS and PSWQ, respectively. Only one ISI item, assessing difficulty falling asleep, was found to be a significant predictor of EPDS and PSWQ scores, and it was more strongly predictive of EPDS score.

Logistic regression assessed if scoring above the moderate and severe ISI cutoffs was associated with increased risk for scoring above the EPDS and PSWQ thresholds. Women who scored above the threshold for moderate insomnia symptoms had significantly greater ORs for reporting significant depression symptoms (OR 7.7, confidence interval [CI] 3.76-15.78, $p<0.001)$ and reporting symptoms of generalized anxiety (OR 2.55, CI 1.39-4.69, $p=0.003$ ) compared to those women who scored below the ISI cutoff. The ORs for reporting symptoms of depression (OR 11.11, CI 1.48-83.37, $p=0.019$ ) and generalized anxiety symptoms (OR 3.59, CI 1.32-9.79, $p=0.012$ ) also were significantly greater for women who scored above the threshold for severe insomnia symptoms.

The effect size for the difference in insomnia symptoms between women who scored above the EPDS and PSWQ was also computed using Cohen's $d$. These results are shown in Table 5. There was a large effect for insomnia symptoms on depressive symptoms and a moderate effect on generalized anxiety symptoms.

Table 4. Insomnia Severity Index Items as Predictors of Penn State Worky Questionnaire Total SCORE

\begin{tabular}{lcccc}
\hline & $B$ & $S E B$ & $\beta$ & $\mathrm{p}$ \\
\hline Maternal age & -0.302 & 0.19 & -0.09 & 0.105 \\
Difficulty falling asleep & 2.56 & 1.18 & 0.17 & 0.031 \\
Difficulty staying asleep & 0.63 & 1.32 & 0.04 & 0.635 \\
Problem waking too early & 1.29 & 1.00 & 0.09 & 0.197 \\
Dissatisfaction with sleep pattern & 0.64 & 1.55 & 0.03 & 0.680 \\
Daytime functioning impaired & 0.79 & 1.44 & 0.05 & 0.582 \\
Impairment noticeable to others & 1.32 & 1.16 & 0.09 & 0.255 \\
Distress about sleep problem & 1.40 & 1.38 & 0.09 & 0.309 \\
\hline
\end{tabular}

Table 5. Comparison of Mean Insomnia Severity Index Scores for Women Above and Below Edinburgh Postnatal Depression Scale and Penn State WorRy QUESTIONNAIRE THRESHOLDS

\begin{tabular}{ccccc}
\hline & $\begin{array}{c}E P D S<14 \\
\mathrm{n}=61\end{array}$ & $\begin{array}{c}E P D S \geq 14 \\
\mathrm{n}=187\end{array}$ & $\mathrm{t}$ & Cohen's d \\
\hline $\begin{array}{c}\text { ISI score } \\
\text { mean } \pm \text { SD }\end{array}$ & $9.49 \pm 4.97$ & $15.86 \pm 5.77$ & -7.73 & 1.14 \\
& $\begin{array}{c}P S W Q<60 \\
\mathrm{n}=96\end{array}$ & $\begin{array}{c}P S W Q \geq 60 \\
\mathrm{n}=153\end{array}$ & & \\
& $11.67 \pm 5.95$ & $15.92 \pm 5.81$ & -5.58 & 0.72 \\
\hline $\begin{array}{c}\text { ISI score } \\
\text { mean } \pm \mathrm{SD}\end{array}$ & & & & \\
\hline
\end{tabular}

All $p \leq 0.001$.

\section{Discussion}

More than half of the participants in this sample of 257 perinatal women who sought psychiatry outpatient treatment reported moderately severe insomnia symptoms, and $12 \%$ reported severe insomnia symptoms. As expected in this population, clinically significant depression symptoms were present in $75 \%$ of the participants, and $61 \%$ had clinically significant generalized anxiety symptoms. Mean scores and proportions of women scoring above the clinical threshold on the measures were comparable between pregnant and postpartum women.

In this relatively large sample of treatment-seeking perinatal women, insomnia symptoms were linked to depression and generalized anxiety symptoms. However, the relationships among insomnia symptoms, depression symptoms, and generalized anxiety symptoms are not straightforward and in this sample relate to whether or not the woman is pregnant or postpartum. Specifically, among pregnant women, there was no relationship between depression symptoms and insomnia symptoms when controlling for the variance associated with generalized anxiety symptoms. Among postpartum women, this relationship remained even after controlling for generalized anxiety symptoms. Alternatively, the relationship between generalized anxiety symptoms and insomnia symptoms, when controlling for depression symptoms, was small but significant for pregnant women but not for postpartum women. There was no relationship between generalized anxiety symptoms and insomnia symptoms for postpartum women when controlling for depression symptoms. The results suggest that although there is shared variance for depression and generalized anxiety symptoms in relation to insomnia symptoms, the unique contribution of the two symptom clusters is different for pregnant and postpartum women. Therefore, these symptom clusters may be contributing to insomnia symptoms in different ways. It appears that for pregnant women, anxiety symptoms are the larger contributor to insomnia symptoms; whereas for postpartum women, depression symptoms are the larger contributor.

Women who had moderate symptoms of insomnia had 7-fold greater odds of scoring above the threshold for depressive symptoms and 2-fold greater odds of scoring above the threshold for generalized anxiety symptoms. These ORs are similar to those found in a population-based study of postpartum women examining sleep quality and depression. ${ }^{13}$ 
ORs for experiencing depression symptoms were even greater among women who reported severe insomnia symptoms (11-fold); however, ORs for experiencing generalized anxiety symptoms were comparable relative to women who reported moderate insomnia symptoms. This suggests that although generalized anxiety symptoms do not change with insomnia symptom severity, depression symptoms are significantly influenced by insomnia symptom severity.

Correspondingly, large and moderate effects were found for insomnia symptoms between women scoring above and below the EPDS and PSWQ thresholds, respectively. Although ISI total score was clearly related to both depression and anxiety measures, when examining the contribution of each item to these measures, only difficulty falling asleep was a significant predictor of depression and anxiety scores. This finding is consistent with Goyal et al.'s results, which showed that delayed sleep onset was most strongly associated with depressive symptoms. ${ }^{11}$

There are several limitations of this study that warrant discussion. The only demographic variable available in our dataset was maternal age. It is likely that other demographic variables and psychosocial factors, such as parity, socioeconomic status, marital or partnered status, psychiatric history, and social support, influence the relationship between sleep and mood. Our methods of characterizing insomnia, depression, and generalized anxiety symptoms, although practical for a large sample, have limitations. The ISI and PSWQ have not been validated in childbearing samples, and the PSWQ only measures symptoms of generalized anxiety, which may limit the generalizability of the findings to other anxiety symptoms. Clinician-rated assessments of mood and objective measures of sleep would allow more comprehensive characterization of psychiatric symptoms and sleep patterns. Lack of information about history of sleep problems and if external causes (e.g., caring for an infant, physical discomfort) produced the sleep difficulties is also a limitation. Our sample consisted of treatment-seeking perinatal women, and these results may not generalize to perinatal women who are not at risk for mental health problems. Finally, the cross-sectional design does not allow for conclusions about causality.

\section{Conclusions}

The findings from this study have significant implications for clinical practice. Considering that more than half of the perinatal women who came for psychiatric outpatient treatment reported clinically significant symptoms of insomnia, all perinatal women seen in these settings should be routinely assessed for sleep problems. There are few self-report sleep measures that are validated for use in perinatal women, and validation of the ISI in childbearing women is important. Modifying the ISI so that only those sleep disturbances most likely to represent insomnia (e.g., instructing women to indicate whether they experience these symptoms unrelated to infant care or environmental disturbance) would better tailor the measure to this population.

Our results suggest that asking just one sleep-related question (e.g., about difficulty falling asleep) may help identify women who are at risk for experiencing significant mood problems related to insomnia. The optimal treatment approach for sleep disturbance may vary depending on whether the woman is pregnant or postpartum. In our sample, anxiety was a strong contributor to the relationship between mood and insomnia for pregnant but not postpartum women. Therefore, treatment of anxiety in pregnant women who are reporting insomnia may lead to sleep improvements. Interventions targeting insomnia are critical for this population, although more research is needed to determine if sleep interventions in this population cause improvements in symptoms of depression and anxiety. Studies using longitudinal methodologies (e.g., following women from before conception through the first 6 months postpartum) will be essential to fully appreciate the nature of the relationships between sleep and mental health around the time of childbearing.

\section{Acknowledgments}

We thank Allison Roberts for her work in organizing the study database.

\section{Disclosure Statement}

No competing financial interests exist.

\section{References}

1. Vesga-Lopez O, Blanco C, Keyes K, Olfson M, Grant BF, Hasin DS. Psychiatric disorders in pregnant and postpartum women in the United States. Arch Gen Psychiatry 2008; 65:805-815.

2. Gavin NI, Gaynes BN, Lohr KN, Meltzer-Brody S, Gartlehner G, Swinson T. Perinatal depression: A systematic review of prevalence and incidence. Obstet Gynecol 2005;106: 1071-1083.

3. Matthey S, Barnett B, Howie P, Kavanagh DJ. Diagnosing postpartum depression in mothers and fathers: Whatever happened to anxiety? J Affect Disord 2003;74:139-147.

4. Wenzel A, Haugen E, Jackson L, Brendle J. Anxiety symptoms and disorders at eight weeks postpartum. I Anxiety Disord 2005;19:295-311.

5. Hollins K. Consequences of antenatal mental health problems for child health and development. Curr Opin Obstet Gynecol 2007;19:568-572.

6. Maughan A, Cicchetti D, Toth SL, Rogosch FA. Earlyoccurring maternal depression and maternal negativity in predicting young children's emotion regulation and socioemotional difficulties. J Abnorm Child Psychol 2007;35:685703.

7. Goodman SH, Gotlib IH. Risk for psychopathology in the children of depressed mothers: A developmental model for understanding mechanisms of transmission. Psychol Rev 1999;106:458-490.

8. Lee KA, Zaffke ME, McEnany G. Parity and sleep patterns during and after pregnancy. Obstet Gynecol 2000; 95:14-18.

9. Bei B, Milgrom J, Ericksen J, Trinder J. Subjective perception of sleep, but not its objective quality, is associated with immediate postpartum mood disturbances in healthy women. Sleep 2010;33:531-538.

10. Montgomery-Downs HE, Insana SP, Clegg-Kraynok MM, Mancini LM. Normative longitudinal maternal sleep: The first 4 postpartum months. Am J Obstet Gynecol 2010;203: 465.e1-465.e7.

11. Goyal D, Gay C, Lee K. Patterns of sleep disruption and depressive symptoms in new mothers. I Perinat Neonatal Nurs 2007;21:123-129. 
12. Skouteris H, Germano C, Wertheim E, Paxton S, Milgrom J. Sleep quality and depression during pregnancy: A prospective study. J Sleep Res 2008;17:217-220.

13. Dørheim S, Bondevik G, Eberhard-Gran M, Bjorvatn B. Sleep and depression in postpartum women: A population-based study. Sleep 2009;32:847-855.

14. Okun ML, Luther J, Prather AA, Perel JM, Wisniewski S, Wisner KL. Changes in sleep quality, but not hormones predict time to postpartum depression recurrence. J Affect Disord August 11, 2010 [Epub ahead of print].

15. Bastien C, Vallires A, Morin C. Precipitating factors of insomnia. Behav Sleep Med 2004;2:50-62.

16. Jefferson $C$, Drake $C$, Scofield $H$, et al. Sleep hygiene practices in a population-based sample of insomniacs. Sleep 2005;28:611-615.

17. Lee KA, Caughey AB. Evaluating insomnia during pregnancy and postpartum. In: Attarian HP, ed. Current clinical neurology: Sleep disorders in women. Totowa, NJ: Humana Press, 2006:185.

18. Krystal AD. Depression and insomnia in women. Clin Cornerstone 2004;6 (Suppl 1B):S19-28

19. Breslau N, Roth T, Rosenthal L, Andreski P. Sleep disturbance and psychiatric disorders: A longitudinal epidemiological study of young adults. Biol Psychiatry 1996;39:411-418.
20. Buysse D, Angst J, Gamma A, Ajdacic V, Eich D, Rssler W. Prevalence, course, and comorbidity of insomnia and depression in young adults. Sleep 2008;31:473-480.

21. Bastien $\mathrm{CH}$, Vallires A, Morin CM. Validation of the Insomnia Severity Index as an outcome measure for insomnia research. Sleep Med 2001;2:297-307.

22. Cox J, Holden J, Sagovsky R. Detection of postnatal depression. Development of the 10-item Edinburgh Postnatal Depression Scale. Br J Psychiatry 1987;150:782786.

23. Cox JL, Chapman G, Murray D, Jones P. Validation of the Edinburgh Postnatal Depression Scale (EPDS) in non-postnatal women. J Affect Disord 1996;39:185-189.

24. Meyer TJ, Miller ML, Metzger RL, Borkovec TD. Development and validation of the Penn State Worry Questionnaire. Behav Res Ther 1990;28:487-495.

Address correspondence to: Leslie M. Swanson, Ph.D. 4250 Plymouth Road Ann Arbor, MI 28109

E-mail:1mswan@med.umich.edu 